\title{
Declaração de Deli: Alma-Ata revisitada. Tradução portuguesa
}

José Augusto Simões, ${ }^{1}$ Inês Madanelo, ${ }^{2}$ Tiago Mendes ${ }^{3}$

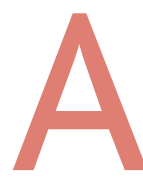

15a Conferência Mundial sobre Saúde Rural daWONCA, realizada em Nova Deli, India, de 26 a 29 de abril de 2018, sobre o lema HEALING THE HEART OF HEALTHCARE - LEAVING NO ONE BEHIND, aprovou a Declaração de Deli: Alma-Ata revisitada. ${ }^{1}$

Este artigo apresenta a tradução para português dessa Declaração, efetuada pelos autores, membros portugueses da EURIPA (The European Rural and Isolated Practitioners Association), cujo International Advisory Board (EURIPA-IAB) incentivou os seus membros a traduzirem e publicarem na língua materna a Declaração de Deli.

Esta Declaração revisita a Declaração de Alma-Ata de $1978^{2}$ e apela às Nações Unidas, aos seus comités especializados e aos governos dos diferentes países para que continuem a esforçar-se até alcançar as metas estabelecidas há 40 anos.

A Declaração de Alma-Ata definiu a saúde como "um estado de completo bem-estar físico, mental e social, e não meramente a ausência de doença ou enfermidade". ${ }^{2}$ Os profissionais de saúde que atuam em contexto rural procuram basear o seu trabalho nesse princípio e esforçam-se para obter o mais alto nível de saúde possível para as comunidades que servem, permitindo ambicionar o objetivo de uma "saúde para todas as pessoas que vivem no mundo rural". ${ }^{3}$

As estratégias globais sobre recursos humanos para a saúde da Organização Mundial da Saúde reforçam a necessidade de trabalhadores da saúde com responsabilidade social. ${ }^{4-5}$ Neste contexto, as necessidades das pessoas que vivem nas áreas rurais e isoladas do mundo devem merecer que as nações alcancem uma cobertura universal em saúde, "não deixando ninguém para trás". ${ }^{3}$

No espírito de Alma-Ata, a Declaração de Deli identifica seis áreas prioritárias: ${ }^{3}$

1. Médico de Família. UCSP Mealhada, ACeS Baixo Mondego. EURIPA-IAB. 2. Médica Interna de Medicina Geral e Familiar. UCSP Vouzela, ACeS Dão-Lafões. 3. Médico de Família. USF Matriz, ACeS Alentejo Central.
- Equidade e acesso aos cuidados - A verdadeira cobertura universal de saúde só será alcançada quando as pessoas nas zonas rurais do mundo tiverem equidade de acesso aos cuidados de saúde que supere as atuais barreiras geográficas, sociais, económicas, políticas, étnicas e culturais. ${ }^{6}$

- Presença rural na política-As vozes das pessoas das zonas rurais precisam ser ouvidas no desenvolvimento de políticas que os afetam. ${ }^{7}$

- Desenvolvimento do sistema de saúde-Um sistema de saúde que apresenta cuidados de saúde primários abrangentes é o mais eficaz e eficiente em termos de custos e resultados de saúde. ${ }^{8}$

- Desenvolver e educar uma capacidade de trabalho adequada à finalidade - Existe evidência clara que recrutar estudantes de áreas rurais, proporcionar aos estudantes experiência rural substancial durante a formação pré-graduada e implementação de programas específicos de capacitação rural no período pós-graduado são as intervenções eficazes para conseguir o recrutamento e cativação para zonas rurais.9.11

- Redirecionando a investigação - A investigação e a construção de evidência em contexto rural devem ser expandidas para fundamentar a tomada de decisão dos governos sobre os serviços de saúde rurais. ${ }^{12}$

- Pessoas e comunidades - A população rural é digna de sistemas de saúde sensíveis às suas culturas, idiomas e tradições. As populações rurais estão mais envelhecidas do que as urbanas e, consequentemente, têm um maior número de indivíduos vulneráveis. $\mathrm{O}$ peso do estado de doença, sobretudo o decorrente de doenças crónicas, aumenta a pressão sobre os sistemas de saúde rurais. ${ }^{13}$

Desenvolver os cuidados de saúde primários e a medicina rural para todas as populações rurais é sinónimo de cumprimento das aspirações de Alma-Ata e de uma cobertura universal de Saúde, priorizando a saúde rural em termos de profissionais de saúde e de infraestruturas capazes de eliminar as desigualdades existentes. ${ }^{5,14}$ 


\section{REFERÊNCIAS BIBLIOGRÁFICAS}

1. WONCA. Rural Round Up: Delhi WONCA Rural conference report. WONCA News. 2018;44(5):4-6.

2. OMS. Declaração de Alma-Ata: conferência internacional sobre cuidados de saúde primários, Alma-Ata, URSS; 6-12 September 1978 [Internet].Available from: http://cmdss2011.org/site/wp-content/uploads/ 2011/07/Declara\%C3\%A7\%C3\%A3o-Alma-Ata.pdf

3. WONCA Rural Health. The Delhi Declaration: Alma Ata revisited [Internet]. Available from: http://www.globalfamilydoctor.com/site/DefaultSite/filesystem/documents/Groups/Rural\%20Practice/18\%20Delhi\%20Dec.pdf

4. High-Level Commission on Health Employment and Economic Growth. Working for health and growth: investing in the health workforce [Internet]. Geneva:World Health Organization; 2016. ISBN 9789241511308

5. Almeida MM, Floss M, Targa LV, Wynn-Jones J, Chater AB. It is time for rural training in family medicine in Brazil! Rev Bras Med Fam Comunidade. 2018;13(40):1-4.

6. Remoaldo PC. Acessibilidade aos cuidados primários de saúde dos concelhos de Guimarães e de Cabeceiras de Basto [Accessibility to primary health care in Guimarães and Cabeceiras de Basto]. Rev Port Clin Geral 2003;19(2):107-19. Portuguese

7. Ruiz ENF, Gerhardt TE. Políticas públicas no meio rural: visibilidade e participação social como perspectivas de cidadania solidária e saúde [Public policies in rural áreas: visibility and social participation as perspectives of supportive citizenship and health]. Physis Rev Saúde Colet. 2012;22(3):1191-209. Portuguese

8. Organização Mundial da Saúde. Cuidados de saúde primários - Agora mais que nunca: relatório mundial de saúde 2008. Lisboa: Alto Comissariado da Saúde; 2009. ISBN 9789241563734

9. WONCA Working Party on Rural Practice. Política de formação para a prática rural. Rev Bras Med Fam Comunidade. 2013;8(Suppl 1):25-34.
10. Hespanhol AP, Malheiro A, Silva A, Veiga MG, Pereira IA, Ramos JF, et al. Ensino da medicina geral e familiar na Faculdade de Medicina do Porto. Educ Med. 2003;1(1):45.

11. Zorzi A, Rourke J, Kennard M, Peterson M, Miller K. Combined research and clinical learning make rural summer studentship program a successful model. Rural Remote Health. 2005;5(4):401.

12. Kelly L, Rourke J. Research electives in rural health care. Can Fam Physician 2002;48:1476-80.

13. Burille A, Gerhardt TE. Doenças crônicas, problemas crônicos: encontros e desencontros com os serviços de saúde em itinerários terapêuticos de homens rurais [Chronic diseases, chronic problems: agréments and disagreements with health services in therapeutic itineraries of rural men]. Saude Soc. 2014;23(2):664-76. Portuguese

14. Carvalho VK, Marques CP, Silva EN. Mais Médicos (More Doctors) Program: its contribution in view of WHO recommendations for provision of doctors. Ciênc Saúde Colet. 2016;21(9):2773-84.

\section{CONFLITOS DE INTERESSE}

Os autores declaram não possuir quaisquer conflitos de interesse.

\section{ENDEREÇO PARA CORRESPONDÊNCIA}

José Augusto Simões

UCSP Mealhada, ACeS Baixo Mondego - ARS Centro

Rua da Misericória, n 12, 3050-387 Mealhada, Portugal

Telef.: 351231202217

Fax: +351231203502

htp://orcid.org/0000-0003-2264-7086

Recebido em 12-09-2018

Aceite para publicação em 01-10-2018 


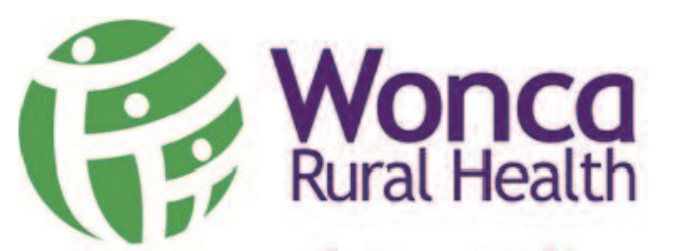

The Wonca Working

Party on Rural Practice

\section{Declaração de Deli \\ Alma-Ata revisitada}

World Organization of Family Doctors World Rural Health Conference, abril 2018

Os participantes na $15^{\text {th }}$ WONCA World Rural Health Conference convidam a comunidade internacional a reafirmar os princípios da Declaração de Alma-Ata. Apela-se às Nações Unidas, respetivos comités especializados e governos nacionais para que continuem a esforçar-se até alcançar as metas estabelecidas há 40 anos.

A Declaração de Alma-Ata definiu a saúde como "um estado de completo bem-estar físico, mental e social, e não meramente a ausência de doença ou enfermidade". Como profissionais de saúde em contexto rural, baseamos o nosso trabalho nesse princípio. Esforçamo-nos para obter o mais alto nível de saúde possível para as comunidades que servimos, com o objetivo de alcançar «Saúde para todas as populações rurais».

Reconhecemos que:

- A concretização deste objetivo requer a ação de outros setores sociais e económicos, para além do setor da saúde: a saúde e o bem-estar são impulsionados tanto pelas condições socioeconómicas quanto pelos cuidados de saúde;

- Os cuidados de saúde primários nas áreas rurais e outras áreas devem ser prestados por equipas de profissionais de saúde com um vasto leque de competências e níveis de diferenciação, trabalhando em parceria com as comunidades;

- As equipas de profissionais de saúde devem focar-se em prestar assistência integral e abrangente que responda às necessidades de indivíduos, famílias e comunidades, afastando-se de um modelo médico fechado e com um pensamento compartimentado;
- O trabalho dos médicos de família, no máximo do seu potencial, desempenha um papel importante na prestação de cuidados centrados na pessoa e na comunidade em áreas rurais;

- As populações rurais estão envelhecidas, mais pobres e mais vulneráveis. Na era dos OBjetivos DE DESENVOLVIMENTO SuSTENTÁVEL devemos promover a equidade na acessibilidade à saúde e assegurar que ninguém fica em desvantagem.

A estratégia global sobre recursos humanos para a saúde da Organização Mundial da Saúde - Workforce 2030 e High-Level Commission on Health Employment and Economic Growth - reforça a necessidade de uma força de trabalho em saúde expandida e com mais responsabilidade social. Além disso, a Declaração de Dublin sobre Recursos Humanos para a Saúde: Construindo a Capacidade de Trabalho de Saúde do Futuro, enfatiza:

"A importância fundamental de uma capacidade de trabalho social e de saúde competente, capacitada, organizada e distribuída de forma otimizada, especialmente em áreas rurais e com baixa acessibilidade, para o fortalecimento do desempenho e resiliência do sistema de saúde".

Neste contexto, acreditamos que as necessidades das pessoas que vivem em áreas rurais e isoladas do mundo devem receber prioridade especial para que as nações alcancem uma cobertura universal de saúde.

No espírito de Alma-Ata, estamos empenhados em trabalhar com a comunidade internacional para al- 
cançar «Saúde para todas as populações rurais». Identificamos como prioritárias as seguintes seis áreas:

Equidade e acesso aos cuidados. A verdadeira cobertura universal de saúde só será alcançada quando as pessoas nas zonas rurais do mundo tiverem equidade de acesso aos cuidados de saúde que supere as atuais barreiras geográficas, sociais, económicas, políticas, étnicas e culturais. Apesar de quase $50 \%$ da população mundial viver em áreas rurais, apenas 38\% dos enfermeiros e $24 \%$ dos médicos do mundo estão alocados a estas regiões. Essa desigualdade/iniquidade deve desaparecer. As populações rurais merecem a mesma qualidade de atendimento em saúde que as populações urbanas - disponível, economicamente acessível e efetiva/eficiente, que responda às necessidades em saúde da população. De acordo com a Organização Internacional do Trabalho, a dicotomia rural-urbano é omnipresente, desde os países mais ricos até aos países mais pobres. Em todo o mundo (174 países), 56\% dos que vivem em zonas rurais não estão cobertos por cuidados básicos de saúde contra $22 \%$ nas cidades e vilas. Questões de género, culturais, etárias e geográficas são primordiais para alcançar a equidade em cuidados de saúde para as populações rurais.

Presença rural na política. As políticas que afetam a saúde e determinam os cuidados de saúde fornecidos às populações rurais são frequentemente desenvolvidas nos centros de poder urbanos. Estes, muitas vezes, não têm em consideração as necessidades das populações rurais ou as implicações para essas pessoas das políticas aplicadas. É necessária investigação mais alargada. As vozes das pessoas de zonas rurais precisam ser ouvidas no desenvolvimento de políticas que os afetam. Um processo de avaliação do impacto sobre a saúde rural das medidas implementadas ou «Presença Rural nas Políticas de Saúde» mitiga os possíveis efeitos negativos para a saúde do desenvolvimento de políticas fragmentadas.

Desenvolvimento do sistema de saúde. Um sistema de saúde que apresenta cuidados de saúde primários abrangentes é o mais eficaz e eficiente em termos de custos e resultados de saúde. Neste contexto, os governos devem investir na expansão dos seus sistemas de saúde com a criação de equipas multidisciplinares de profissionais de saúde, capacitadas para responder às necessidades específicas das comunidades rurais e isoladas. As novas tecnologias emergentes desempenharão um papel cada vez mais importante na sustentação da capacidade de trabalho em saúde e na saúde das comunidades rurais e isoladas. Apelamos à comunidade internacional para que invista em saúde digital e educação em técnicas de saúde digital como suporte de uma capacidade de trabalho em saúde rural adequadamente capacitada.

Desenvolver e educar uma capacidade de trabalho adequada à finalidade. A evidência é clara: recrutar estudantes de áreas rurais, proporcionar aos estudantes experiência rural substancial durante a formação pré-graduada e implementação de programas específicos de capacitação rural no período pós-graduado são as intervenções mais eficazes para conseguir o recrutamento e cativação para zonas rurais.

As instituições de formação em saúde em contexto rural devem desenvolver currículos socialmente responsáveis, assumindo um compromisso de envolvimento comunitário permanente. $\mathrm{O}$ isolamento populacional e a ruralidade impõem desafios adicionais aos profissionais de saúde em contexto rural para conseguirem prestar serviços localmente necessários. Os cuidados de saúde rurais precisam, portanto, de médicos generalistas e médicos de família que estejam adequadamente capacitados e habilitados para o desempenho de uma extensa e abrangente gama de competências para enfrentar os desafios específicos patentes no seu trabalho diário. Por outro lado, a utilização nestas zonas de profissionais de saúde recém-formados e sem capacitação apropriada ao desempenho de cuidados de saúde em zonas rurais isoladas coloca doentes em risco e dissuade os jovens profissionais da escolha de uma carreira profissional em saúde rural. Os profissionais de saúde que trabalham em comunidades rurais devem ter um ambiente de trabalho e condições de vida seguros e justos.

Redirecionando a investigação. É necessária uma ação urgente para reverter a desigualdade na distribuição da investigação «90/10»: menos de 10\% dos recur- 
sos mundiais para investigação em saúde são dedicados a $90 \%$ da mortalidade evitável em países de baixo e médio rendimento. Essa desigualdade é ainda maior em zonas rurais. A investigação e a construção de evidência em contexto rural devem ser expandidas para fundamentar a tomada de decisão do governo sobre os serviços de saúde rurais.

Pessoas e comunidades. A população rural é digna de sistemas de saúde sensíveis às suas culturas, idiomas e tradições. As populações rurais estão mais envelhecidas do que as urbanas e, consequentemente, têm um maior número de indivíduos vulneráveis. O peso do estado de doença, sobretudo o decorrente de doenças crónicas, aumenta a pressão sobre os frágeis sistemas de saúde rurais. A declaração original de Alma-Ata enfatiza que a saúde e o bem-estar não dependem ape- nas dos serviços de saúde, mas também de fatores económicos e sociais. Apelamos aos governos e intervenientes políticos que invistam em infraestruturas, serviços e na economia das áreas rurais. Esta intervenção conjunta terá um impacto duradouro no bem-estar das populações rurais.

Saúde para todas as populações rurais é sinónimo das aspirações de Alma-Ata e da Cobertura Universal de Saúde. Apelamos às Nações Unidas, aos seus comités especializados e aos governos nacionais para que priorizem a saúde rural, os profissionais de saúde e uma infraestrutura capaz a fim de eliminar a desigualdade existente e não deixar ninguém em desvantagem.

$15^{\text {th }}$ WONCA World Rural Health Conference Nova Deli, Índia, abril 2018 